\title{
Wearing face masks strongly confuses counterparts in reading emotions
}

\author{
Claus-Christian Carbon ${ }^{1,2,3}$ \\ ${ }^{1}$ Department of General Psychology and Methodology, University of Bamberg, Germany \\ Markusplatz 3, D-96047 Bamberg, Bavaria, Germany \\ email: ccc@uni-bamberg.de; phone: +49 9518631860 \\ ${ }^{2}$ Research Group EPÆG, Bamberg, Bavaria, Germany \\ ${ }^{3}$ Geomedi University, Tbilisi, Georgia
}

This research was not supported by any special funding.

We declare no competing interests.

One sentence summary: When we wear face masks, counterparts cannot read our emotional status accurately and confidently any more.

Word count: 3,600

Running title: The impact of face masks on emotional reading 


\begin{abstract}
(195 words)
Wearing face masks is one of the essential means to prevent the transmission of certain respiratory diseases such as COVID-19. Although acceptance of such masks increases in the Western hemisphere, many people feel that social interaction is affected by wearing a mask. In the present experiment, we tested the impact of face masks on the readability of emotions. The participants $(N=41$, calculated by an a priori power test; random sample; healthy persons of different ages, 18-87 years) assessed the emotional expressions displayed by twelve different faces. Each face was randomly presented with six different expressions (angry, disgusted, fearful, happy, neutral, sad) while being fully visible or partly covered by a face mask. Lower accuracy and lower confidence in one's own assessment of the displayed emotions indicate that emotional reading was strongly irritated by the presence of a mask. We further detected specific confusion patterns, mostly pronounced in the case of misinterpreting disgusted faces as being angry plus assessing many other emotions (e.g. happy, sad and angry) as neutral. We discuss compensatory actions that can keep social interaction effective (e.g. body language, gesture and verbal communication), even when relevant visual information is crucially reduced.
\end{abstract}

Keywords: Emotion, face, masks, accuracy, confusion, occlusion, social psychology, COVID-19 pandemic 


\section{Main text}

Wearing face masks ${ }^{1}$ is indicated in many scenarios, mostly in clinical contexts, when being infected by certain respiratory diseases or in times of epidemics where the risk of potential transmission through air passages has to be reduced (Jefferson et al., 2008). During the COVID19 pandemic (Coronavirus disease 2019), most countries and health organizations like the WHO propagated wearing face masks by early 2020 as a key strategy to reduce the spread of the SARS 2 (Severe Acute Respiratory Syndrome) coronavirus.

Face masks do not only have a direct positive medical impact in terms of preventing the virus from spreading to those who are most vulnerable (Wu \& McGoogan, 2020); they also have positive societal effects as wearing masks allows for relaxing other preventive measures such as strict isolation and quarantining (Mniszewski, Del Valle, Priedhorsky, Hyman, \& Hickman, 2014). However, face masks also cover, per definition, a major part of the human face, which can crucially affect social interaction. Our faces provide the key information of "person identity", "directed visual information" (e.g. attractiveness, age, sex), information supporting the understanding of speech by enabling "facial speech analysis" as well as fine-grained information that allows for reading the other's emotional state via "expression analysis" (Bruce \& Young, 1986). We can compensate a lack of signal for all of these facets of face processing (Grüter \& Carbon, 2010), but often we might reduce the efficacy of processing, the confidence in our assessments and we are susceptible to lose a part of the multichannel-multisensory integration possibilities to cross-check and validate our assessments. Some of these signals that faces provide are processed very fast (identity, Carbon, 2011; gender and attractiveness, Carbon,

\footnotetext{
${ }^{1}$ Face masks show a great variety of forms and technologies; within the present paper, we will focus on masks that look like simple surgical masks and that people can fabricate themselves, so called community masks.
} 
Faerber, Augustin, Mitterer, \& Hutzler, 2018; emotion, Willis \& Todorov, 2006), although the validity of the final assessments are under great dispute (Rojahn, Gerhards, Matlock, \& Kroeger, 2000; Russell, 1994).

With regard to expression analysis, different studies showed that we are far from being perfect in assessing the emotional state of our counterpart just by inspecting the face (Derntl, Seidel, Kainz, \& Carbon, 2009) without knowing the context of a scene (Aviezer et al., 2008) or without information about the dynamic evolvement of the seen expression (Bassili, 1979; Blais, Fiset, Roy, Saumure Régimbald, \& Gosselin, 2017). A partial occlusion of the face (Bassili, 1979), e.g. by sunglasses (Roberson, Kikutani, Doge, Whitaker, \& Majid, 2012) or by scarfs (Kret \& de Gelder, 2012)is a further obstacle to accurately reading emotions from facial expressions (Bassili, 1979). Face masks or community masks, as the ones commonly worn during the COVID-19 pandemic to shield the mouth and the nose, cover about $60-70 \%$ of the area of the face that is relevant for emotional expression and thus emotion reading (e.g. $\sim 65 \%$ in the case of the depicted persons in our face set-exact numbers are hard to tell; we can only rely on rough estimations as indicative face areas differ across persons). Crucially, these masks cover an area of the face that is crucial for the effective nonverbal communication of emotional states. Although specific research on the impact of such face masks on emotional recognition is missing, there are some indications from research on the effect of different kinds of facial occlusions. An important source of data are the so-called "Bubbles"-experiments that make use of a general technique developed by Gosselin and Schyns (2001). This technique allows for identifying the specific visual information that is most relevant to human categorization performance, for instance information needed to express and read emotions. Other paradigms comprise the presentation of top vs. bottom halves of faces (Bassili, 1979) or the partial 
occlusion of target faces with ecological valid items such as a niqāb (Fischer, Gillebaart, Rotteveel, Becker, \& Vliek, 2012), a shawl or a cap (Kret \& de Gelder, 2012) in order to test for differences in the participants' emotion reading performance. These different paradigms operate with very different stimuli and they were used with samples from different populations. Taken together, they do not enable immediate conclusions about the specific impact of face masks on the reading of different emotions. The manipulations realized in those paradigms are, neither quantitatively nor qualitatively, analogous to the actual practical use of face masks. Further, the results of studies that applied these paradigms are often incoherent, sometimes even contradictive. There is, for instance, a relatively high consensus that covering the lower face parts yields reduced performance in assessing a happy emotional state (e.g., Eisenbarth \& Alpers, 2011; Fischer et al., 2012; Kotsia, Buciu, \& Pitas, 2008). For other emotional states, however, there are quite contradictive results to be found in the literature, e.g., for fear detection (in favour of higher relevance of the eyes, see Bombari et al., 2013; in favour of higher relevance of the mouth, see Kotsia et al., 2008). There is even evidence that a partial coverage of the face might lead to better performance due to fading out irrelevant or deceptive information in faces (Kret \& de Gelder, 2012). Laypersons, for instance, were more accurate in detecting deception in persons who wore a niqāb than in persons who did not (Leach et al., 2016). Inconsistent results such as angry faces attracting more attention to the eyes than the mouth (Eisenbarth \& Alpers, 2011) while the occlusion of the mouth resulted in lower accuracy of detecting anger (Kotsia et al., 2008) have to be interpreted with caution as we do not know the causal or temporal interdependence of such processes. Specific types of occlusions might interfere with different emotions: For example, the mouth seems important for the detection of happiness and fear, but the eyes are more relevant for anger, fear, and sadness (Bombari et al., 2013). 
The present study specifically tested how a common face mask, which for instance dominates the social scenes during the COVID-19 pandemic, changes the efficacy of emotion reading expressions displayed by different faces. Besides recognition sensitivity, we were particularly interested in the confusion of certain emotions with other emotional states due to an increase of signal ambiguity in order to understand everyday life problems in effectively communicating when wearing face masks.

\section{Experimental Study}

\section{Methods}

Participants. The needed sample size of $N=36$ was calculated a priori via power analysis (Faul, Erdfelder, Lang, \& Buchner, 2007) targeting a repeated measures Analysis of variance (ANOVA) with 6 groups (emotions) and 2 measurements (mask vs. no mask) and the ability to detect a medium effect size of $f=0.25$ (Cohen, 1988), given an $\alpha=0.05$ and a test power $(1-\beta)=$ 0.80. From our entire set of data from 41 participants $\left(M_{\text {age }}=26.7\right.$ years $[18-87$ years $], N_{\text {female }}=$ 30) we could use all data sets as all participants reached the pre-defined criterion of showing at least a performance of correctly identifying emotional states in $50 \%$ of the cases where faces were presented without masks (actually, the performance was much higher, see results). This slightly higher actual than needed number of participants resulted in an achieved post hoc test power of 0.88 .

Material. All face stimuli were obtained from the MPI FACES database (Ebner, Riediger, \& Lindenberger, 2010) by a study-specific contract effective by 27 April 2020. As base faces on 
which we later applied face masks, we used frontal photos of 12 Caucasians ( 6 female, 6 male) who belonged to three different face age groups (young, medium, elderly) yielding two persons per face se $\times$ face age group cell. For each person, six different pictures were used that showed the emotional states angry, disgusted, fearful, happy, neutral and sad. For the application of face masks to all of these 72 original pictures we photographed a typical homemade (beige)

community mask. The image of the mask was cut out via Photoshop and individually applied to the different face versions. Realistic shadows were added to create maximally realistic and plastic pictures of persons wearing a face mask (Figure 1).
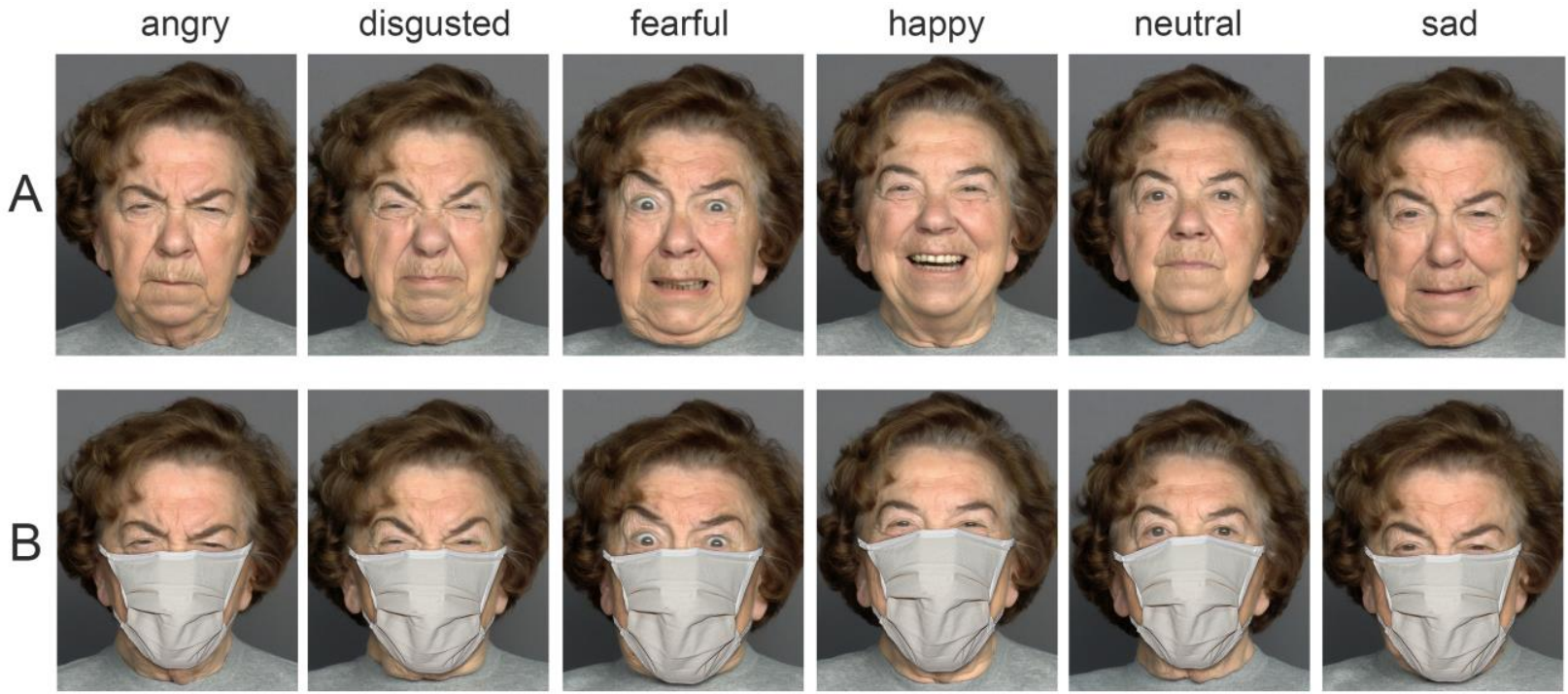

Figure 1: A person showing six different emotions without a mask (A) and wearing a mask $(B)$. Original material from top row stems from MPI FACES database (Ebner et al., 2010).

In sum, we obtained 2 [face sex] $\times 3$ [face age group] $\times 2$ [individuals] $\times 6$ [emotions $] \times 2$ [no face mask vs. face mask] $=144$ face stimuli.

Procedure. The experiment which ran on the SoSciSurvey online platform was conducted between 15 May (10:01 local time) and 18 May (19:45 local time) during the COVID-19 
pandemic when general legal obligations to wear masks in Germany were already in action. Prior to the experimental session, written informed consent was obtained from each participant. All data were collected anonymously. Each participant was exposed to the complete set of stimuli one after another, with the order of stimuli being randomized across participants. Participants were asked to spontaneously assess the depicted person's emotional state from a list of six emotions reflecting the same compilation of emotions shown by the different versions of the faces (angry, disgusted, fearful, happy, neutral and sad). The personal confidence for each assessment had to be indicated on a scale from 1 (very unconfident) to 7 (very confident). There was no time limit for giving a response. The general study design (psychophysical testing) was given ethical approval by the local ethics committee of the University of Bamberg. The entire procedure lasted approximately 20-25 minutes.

\section{Results}

Data were submitted to further data processing executed by R 4.0.0 (R Core Team, 2014), with linear mixed models being analyzed via toolbox lmer (Kuznetsova, Brockhoff, Rune, \& Christensen). The entire, anonymized, data set is available at the Open Science Framework https://osf.io/ka3s6/.

Overall performance for correctly identifying facial emotions in faces without masks was quite remarkable, $M=89.5 \%$ (chance rate $=16.7 \%$ ) with no participant performing below an overall rate of $76.4 \%$. As shown by the mean data for each emotional state (Figure 2), presenting a mask on faces showed a clear performance drop in reading emotions in faces. With the exception of fearful and neutral faces, for which ceiling performance effects were observed, all emotional states were harder to read out from faces with masks. 


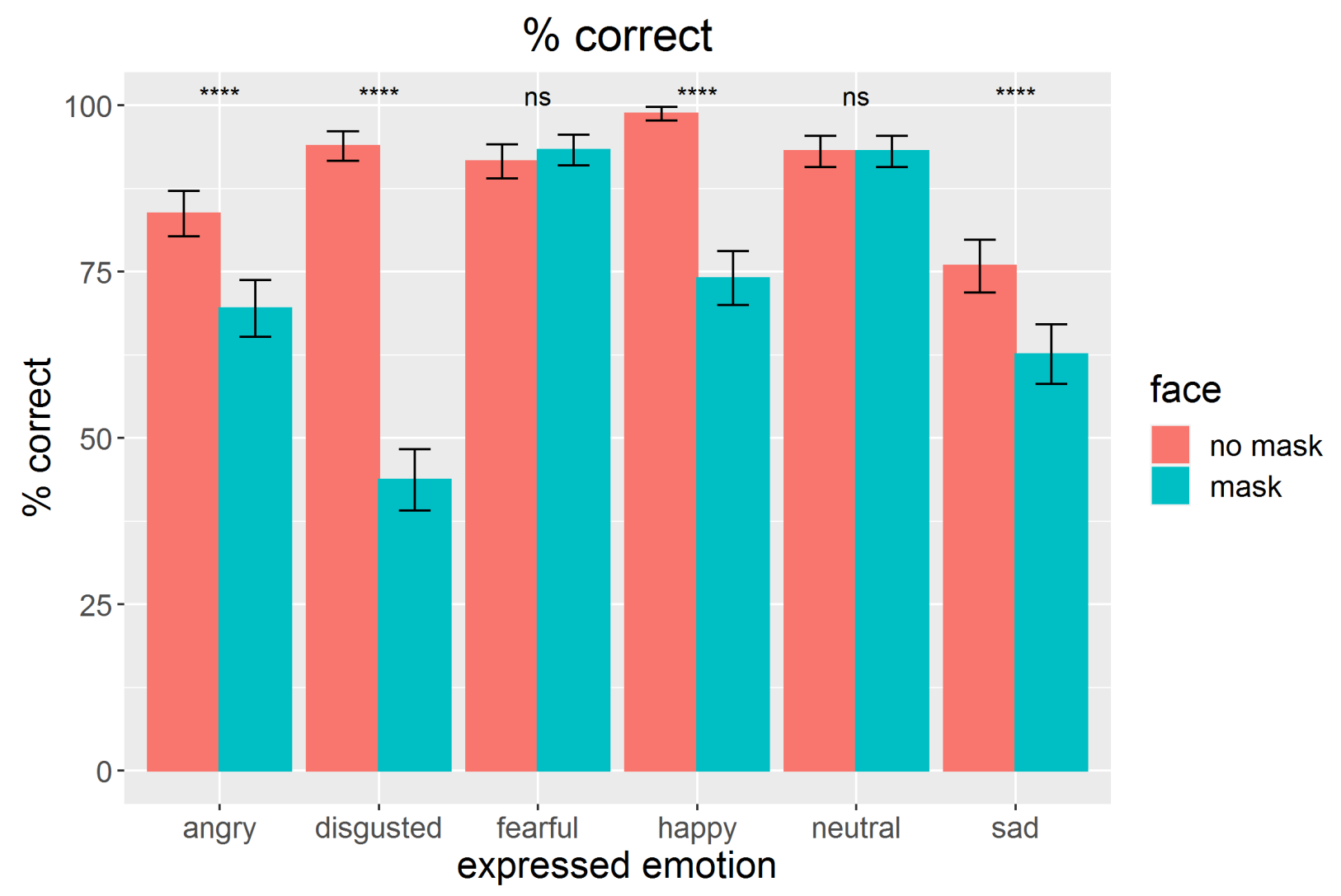

Figure 2: Mean percentage of correct assessment of the emotional states for faces with masks (blue) or without masks (red) on the face. Error bars indicate confidence intervals CI-95\% based on adjusted values for taking within-subjects variances into account (Morey, 2008). Asterisks indicate statistical differences between conditions of wearing and non-wearing on basis of paired t-tests: ****: $p<.0001-n s:$ not significant.

We tested the effect of wearing masks on the performance of emotional reading in faces by means of linear mixed models (LMM) with face mask (face with a mask vs. without a mask) as a fixed factor against a base model (model \#0) which only contained the participants and base stimuli as random intercepts and face emotion as fixed slopes- FS (fixed factors). We furthermore tested in a successive way the effect of the sex and the age group of the face stimuli by adding these factors as $F S$-including all possible interactions of all fixed factors. $P$-values were obtained by likelihood ratio tests of the subsequent models against the respective one-step 
less complex model. The coefficient of determination for each model was calculated via a

likelihood-ratio test utilizing the toolbox MuMIn (Barton, 2019). See Table 1 for detailed results.

Table 1: Linear Mixed Effects analysis of different models in comparison to a simple base model (model \#0), separated by the two tested dependent variables \%correct (percentage of correct emotion classifications) and confidence (for correct emotion classifications). The best fitting model, while being parsimonious, is indicated by bold face. FS - fixed slopes (fixed factors); RSrandom slopes (random factors); $d f$-degrees of freedom; $R^{2}$-coefficient of determination, based on the likelihood-ratio test; $p\left(\chi^{2}\right)$ - probability of accepting a significant effect despite a non-existent difference regarding the more complex vs. the one-step less complex model.

\begin{tabular}{|c|c|c|c|c|c|}
\hline $\begin{array}{r}\text { Dependent variable / } \\
\text { tested model }\end{array}$ & $d f$ & $A I C$ & $\log L i k$ & $R^{2}$ & $p\left(\chi^{2}\right)$ \\
\hline \multicolumn{6}{|l|}{ \%correct } \\
\hline \#0: base (random intercepts) & 9 & 59598 & -29790 & .090 & \\
\hline$\# 1:+$ FS face mask & 15 & 58945 & -29458 & .187 & $<.0001$ \\
\hline$\# 2:$ + FS face sex & 27 & 58850 & -29398 & .203 & $<.0001$ \\
\hline \#3: + FS face age group & 75 & 58465 & -29157 & .266 & $<.0001$ \\
\hline \multicolumn{6}{|l|}{ confidence } \\
\hline \#0: base (random intercepts) & 9 & 16174 & -8078 & .161 & \\
\hline$\# 1:+$ FS face mask & 15 & 15171 & -7571 & .321 & $<.0001$ \\
\hline$\# 2:$ + FS face sex & 16 & 15173 & -7570 & .321 & $.604 n s$ \\
\hline \#3: + FS face age group & 75 & 15021 & -7436 & .358 & $<.0001$ \\
\hline
\end{tabular}

Linear Mixed Effects analysis revealed that both dependent variables were impacted by the factor face mask. Furthermore, face age group played a role in explaining variance of both dependent variables - for face sex, in contrast, we only found an effect for the accuracy of emotion reading.

As face sex as well as face age group were effective in predicting the correctness of reading out the emotional state from faces, Figure 3 shows the differentiated data for the three- 
way interactive effect with face mask. Lower performance in assessing emotions in masked faces were found for most emotions and sex and age groups.

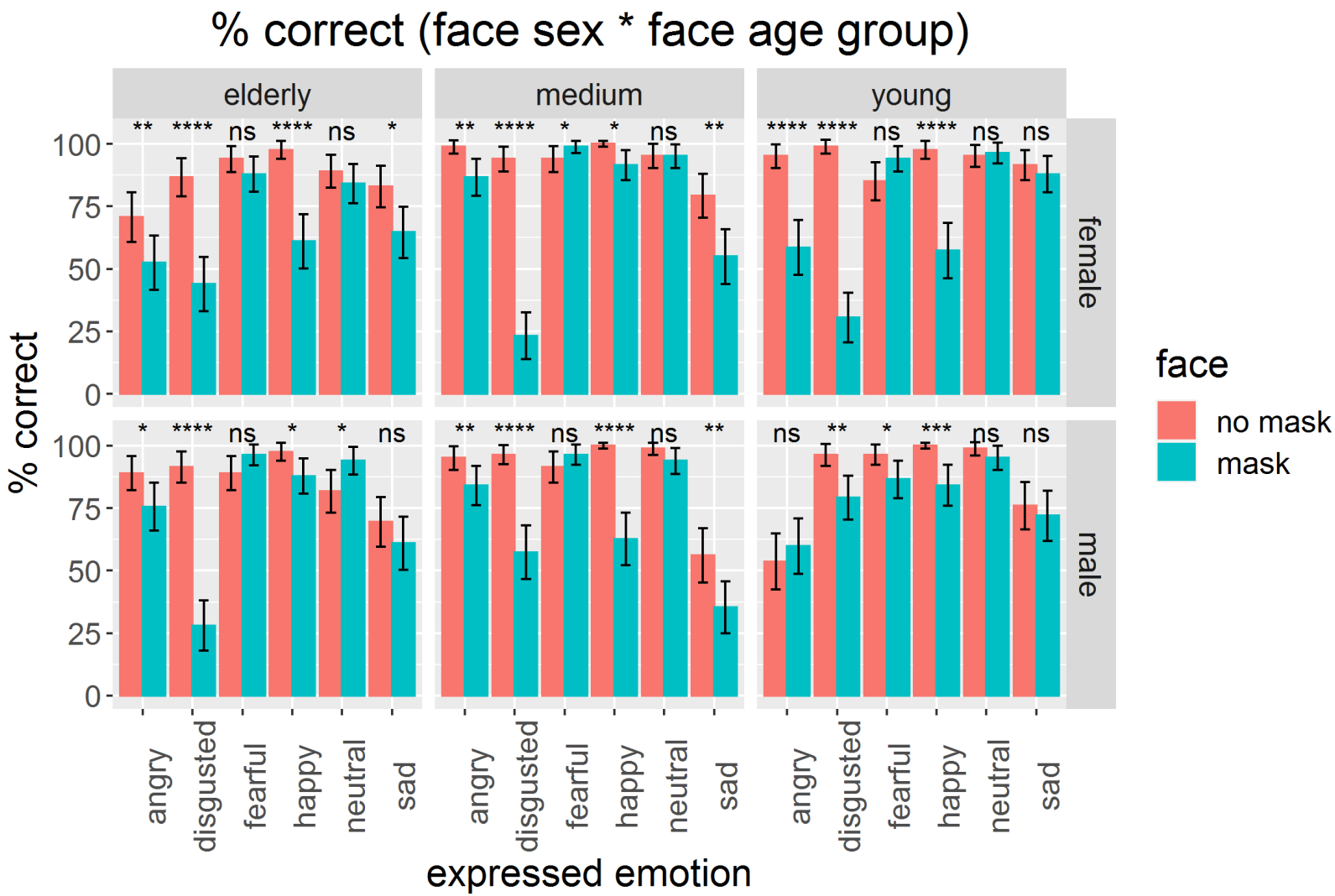

Figure 3: Mean percentage of correctly assessing the emotional states with masks (blue) or without masks (red) on the face, split by face sex and face age group. Error bars indicate confidence intervals CI-95\% based on adjusted values for taking withinsubjects variances into account (Morey, 2008). Asterisks indicate statistical differences between conditions of wearing and nonwearing on basis of paired t-tests: *: p<.05, **: p<.01, ***: $p<.001, * * * *: p<.0001$-ns: not significant.

Based on the finally selected models with face mask, face sex and face age group being included in terms of fixed slopes and their interactions, we obtained several effects of small, medium as well as large size (Table 2). Most importantly, regarding the major question of the study, face mask had a medium-sized effect on the performance of assessing the emotional state of a face and a large-sized effect on the confidence of one's own assessment (for correct emotion classifications). 
Table 2: Statistics of all involved fixed effects terms of the Linear Mixed Effects analysis for the final models (model \#3), separated by the two tested dependent variables \%correct (percentage of correct emotion classifications) and confidence (for correct emotion classifications). $k$ (par)-number of parameters; Cohen's $f$-effect size including qualification as small, medium or large according to (Cohen, 1988)-smaller effects are not further qualified. Note: abbreviated notations for the terms were used to safe space, emotion = face emotion, mask = face mask, sex = face sex, age = face age group .

\begin{tabular}{|c|c|c|c|c|}
\hline & & & $\%$ correct & confidence \\
\hline & term & $k($ par $)$ & Cohen's $f$ & Cohen's $f$ \\
\hline 1 & emotion & 5 & 0.304 medium & 0.263 medium \\
\hline 2 & mask & 1 & 0.253 medium & 0.458 large \\
\hline 3 & sex & 1 & 0.002 & 0.015 \\
\hline 4 & age & 2 & 0.017 & 0.045 \\
\hline 5 & emotion:mask & 5 & 0.263 medium & 0.204 small \\
\hline 6 & emotion:sex & 5 & 0.122 small & 0.060 \\
\hline 7 & mask:sex & 1 & 0.062 & 0.002 \\
\hline 8 & emotion:age & 10 & 0.193 small & 0.159 small \\
\hline 9 & mask:age & 2 & 0.019 & 0.045 \\
\hline 10 & sex:age & 2 & 0.012 & 0.037 \\
\hline 11 & emotion:mask:sex & 5 & 0.059 & 0.055 \\
\hline 12 & emotion:mask:age & 10 & 0.061 & 0.054 \\
\hline 13 & emotion:sex:age & 10 & 0.150 small & 0.095 \\
\hline 14 & mask:sex:age & 2 & 0.047 & 0.032 \\
\hline 15 & emotion:mask:sex:age & 10 & 0.137 small & 0.096 \\
\hline
\end{tabular}


As shown in Figure 4, the confidence data showed a similar but not identical results pattern compared to the percentage of correct assessment data in Figure 2. Interestingly, confidence data reflected the impact of a face mask emotion reading even more clearly. For confidence ratings, also fearful and neutral faces were impacted, probably due to a lack of ceiling effects.

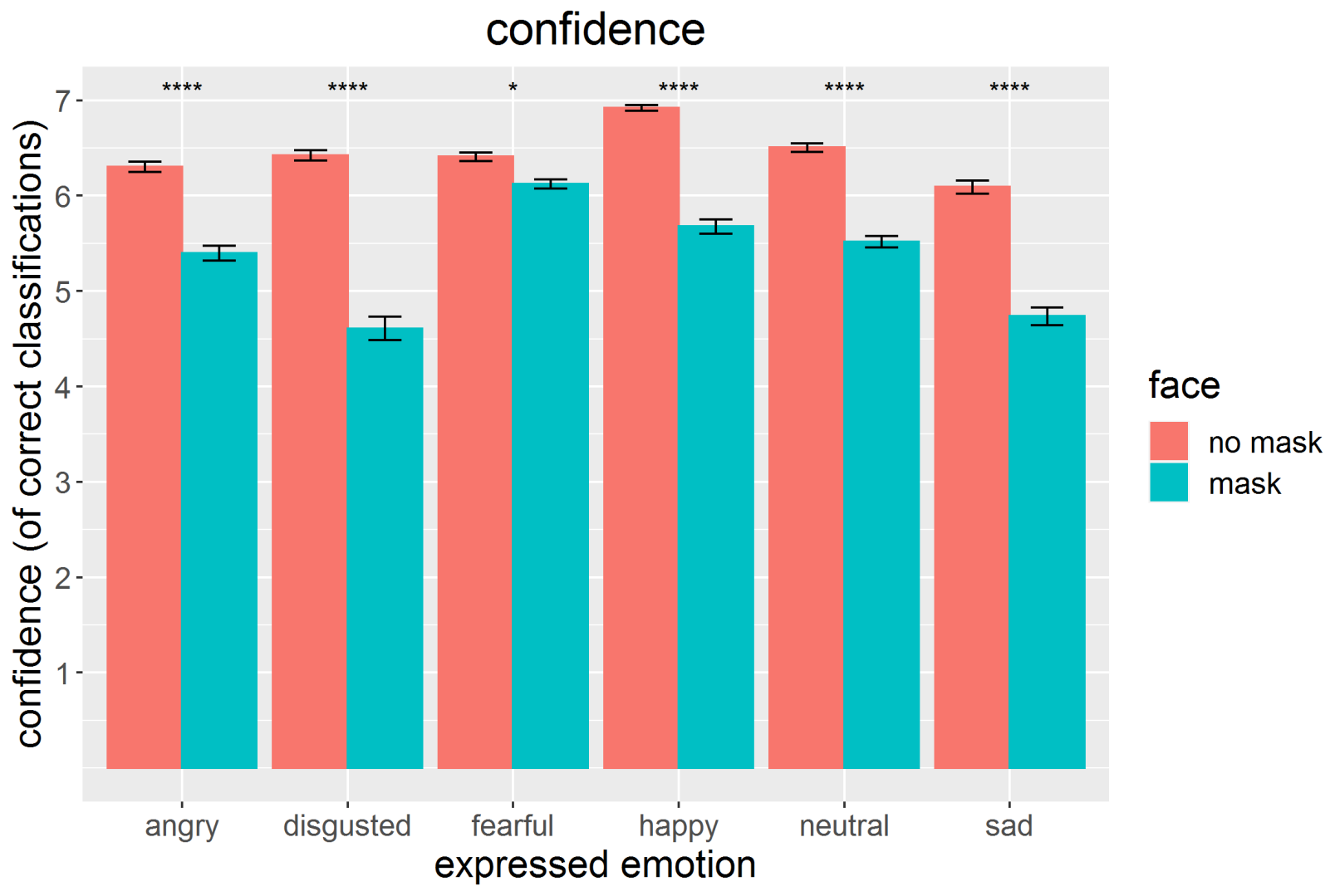

Figure 4: Mean confidence of assessing the emotional states (for correct classifications) with masks (blue) or without masks (red) on the face. Error bars indicate confidence intervals CI-95\% based on adjusted values for taking within-subjects variances into account (Morey, 2008). Asterisks indicate statistical differences between conditions of wearing and non-wearing on basis of paired t-tests: *: $p<.05$, ****: $p<.0001$-ns: not significant.

A drop in performance in reading out emotional states of faces with masks can somehow be expected as hardly any visual information of the lower half of the face is available anymore. To understand how the lack of information is dealt with, it is important to look at the specific 
confusion of individual emotional states — when and in which way are emotions misinterpreted when face masks are worn?

In order to learn about these misinterpretations, we generated confusion matrices for the viewing conditions with faces without masks and with masks (see Figure 5). When faces were shown without masks, the accuracy was much higher as is indicated by clear matches between expressed and perceived emotions. With the exception of the emotional state $s a d$, accuracy was above $83 \%$, but especially sad was often confused with disgusted (20.3\% of the cases). As soon as we applied masks to the faces, this overall very high performance broke down dramatically and characteristic confusions became apparent. For instance, all emotional states with the exception of fearful were repeatedly confused with a neutral state. Sad was often confused with disgusted and neutral, and angry was confused with disgusted, neutral and sad. Most drastically was the misinterpretation of disgusted as angry, which showed up in nearly $38 \%$ of the cases, although such a confusion did only happen in $2 \%$ of the cases when no face mask was used. 


\section{Confusion matrix of emotions}

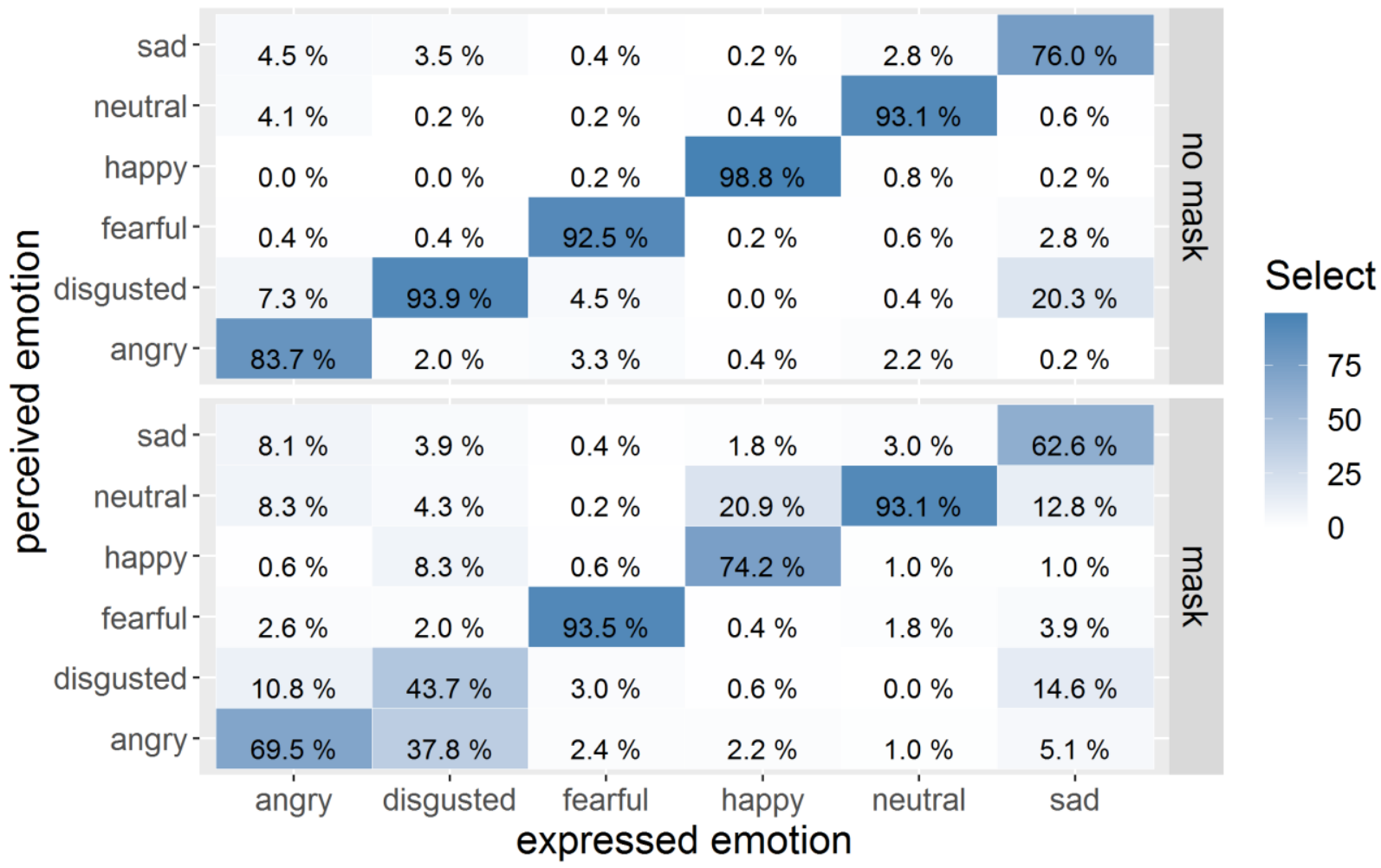

Figure 5: Confusion matrix of expressed and perceived emotions. Top matrix: faces without masks, bottom matrix: faces with a mask. Percentages compile up to 100\% for each expressed emotion. The deeper blue the cell, the higher the score of this cell.

The statistics on the confusion of emotions show clearly how ambiguous an emotional state becomes when an ordinary face mask is worn.

\section{Discussion}

Wearing face masks, even very simple home-made models, is an important measure to effectively decrease the chance of transmitting respiratory diseases (van der Sande, Teunis, \& Sabel, 2008), as is also suggested by the analysis of past pandemics such as the $1918 \mathrm{flu}$ pandemic caused by the H1N1 influenza (Bootsma \& Ferguson, 2007). People in countries where face masks have not been widely used in the past, may still be ambivalent about wearing them. Acquaintance is low, wearing a mask when surrounded by too many non-wearers may feel strange (Carbon, 2020); and for many, there are obvious handling problems and ergonomic 
issues including changed air flow characteristics. Yet, the usage of masks is just becoming an everyday practice here.

In the present experiment, we tested the impact of face masks on emotion reading, which may have important implications for everyday social interaction. We confronted participants with faces showing six different emotions (angry, disgusted, fearful, happy, neutral and sad). The results indicate, that emotion recognition was strongly reduced with the exception of fearful and neutral faces. For fearful faces, as shown before in the literature (but see Bombari et al., 2013; Kret \& de Gelder, 2012; Wegrzyn, Vogt, Kireclioglu, Schneider, \& Kissler, 2017), the eyes region, which was not occluded by the mask, provides most of the emotional information indicative for this emotional state. For neutral faces, the results have to be interpreted in a completely different and cautious way: Although performance for recognizing a neutral state was not directly decreased, many emotional states such as happy, sad and angry were misinterpreted as neutral, so the genuine emotional state was not perceived anymore. Other emotions such as disgusted were confused with angry, and this qualitative misinterpretation which is quite impactful (a person who just does not feel aversion to a very specific thing in a certain situation and who expresses this spontaneously might be interpreted as an angry, potentially aggressive, person) was found in more than one third of all assessments of disgusted faces wearing a mask.

To further qualify these effects, we have to make clear that the face stimuli originated from a scientific database which is aiming to show emotions maximally clear and very pronounced. These requirements were nearly perfectly achieved when we look at the very high performance data for the original faces without masks. There was hardly any confusion of different emotional states (with the exception of sad faces which already showed substantial confounds with disgusted at a level of one fifth of the cases). Such a high performance is hardly 
achievable in everyday life when faces are inspected that show much lower degrees of expressing their emotions by the face. Furthermore, in an everyday life scene we will typically show lower amounts of attention and will invest less time to inspect the face of a counterpart. This means that in natural contexts the impact of face masks on reading emotions could even be stronger. It could further be intensified with increased age: As the results of some empirical studies indicate, older adults have more difficulties recognizing some of the basic emotions (e.g., disgust, happiness, and fear), and even intense problems in recognizing other basic emotions such as anger and sadness (Ruffman, Henry, Livingstone, \& Phillips, 2008).

Face masks may complicate social interaction as they disturb emotion reading from facial expression. This should, however, not be taken as a reason or an excuse for not wearing masks in situations where they are of medical use. We should not forget that humans possess a variety of means to interpret another's state of mind including another's emotional states. Facial expression are not our one and only source of information; we can also take recourse to body posture and body language to infer emotional states of our counterpart. The voice characteristic adds indications from another modality (Golan, Baron-Cohen, \& Hill, 2006), and the social context will provide further information (Mondloch, 2012). Direct verbal communication even helps to understand the very fine-grained state of a mind. We have options - and it is essential to make use of them, not only when being the receiver of socially relevant information, but also when being the sender. Emphasizing alternative communicative channels, we can provide sufficient information to keep going social interaction in a different, yet effective way.

\section{Conflict of Interest Statements}

No conflicts to be reported. 


\section{Acknowledgements}

We would like to thank the MPI for providing the base stimuli for the present study. We also thank our student assistants to have put intensive effort into recruiting many of the participants, and special thanks goes to Christian Meyer for processing the mask stimuli. 


\section{References}

2 Aviezer, H., Hassin, R. R., Ryan, J., Grady, C., Susskind, J., Anderson, A., . . Bentin, S. (2008).

3 Angry, disgusted, or afraid? Studies on the malleability of emotion perception.

$4 \quad$ Psychological Science, 19(7), 724-732. doi:10.1111/j.1467-9280.2008.02148.x

5 Bassili, J. N. (1979). Emotion recognition: The role of facial movement and the relative

6 importance of upper and lower areas of the face. Journal of Personality and Social

7 Psychology, 37(11), 2049-2058. doi:10.1037/0022-3514.37.11.2049

8 Blais, C., Fiset, D., Roy, C., Saumure Régimbald, C., \& Gosselin, F. (2017). Eye fixation

9 patterns for categorizing static and dynamic facial expressions. Emotion, 17(7), 11071119. doi:10.1037/emo0000283

11 Bombari, D., Schmid, P. C., Schmid Mast, M., Birri, S., Mast, F. W., \& Lobmaier, J. S. (2013). Emotion recognition: The role of featural and configural face information. Quarterly Journal of Experimental Psychology, 66(12), 2426-2442.

14
doi:10.1080/17470218.2013.789065

Bootsma, M. C. J., \& Ferguson, N. M. (2007). The effect of public health measures on the 1918 influenza pandemic in US cities. Proceedings of the National Academy of Sciences of the United States of America, 104(18), 7588-7593. doi:10.1073/pnas.0611071104 77(3), 305-327. doi:10.1111/j.2044-8295.1986.tb02199.x

Carbon, C. C. (2011). The first 100 milliseconds of a face: On the microgenesis of early face processing. Perceptual and Motor Skills, 113(3), 859-874. doi:10.2466/07.17.22.Pms.113.6.859-874

Running title: The impact of face masks on emotional reading 
1 Carbon, C. C. (2020). The psychology of wearing face masks in times of the COVID-19 pandemic. Available at SSRN: https://ssrn.com/abstract=3584834. doi:10.2139/ssrn.3584834

Carbon, C. C., Faerber, S. J., Augustin, M. D., Mitterer, B., \& Hutzler, F. (2018). First gender, then attractiveness: Indications of gender-specific attractiveness processing via ERP onsets. Neuroscience Letters, 686, 186-192. doi:10.1016/j.neulet.2018.09.009

Cohen, J. (1988). Statistical power analysis for the behavioral sciences (2nd ed.). Hillsdale, NJ: Lawrence Erlbaum Associates.

Derntl, B., Seidel, E. M., Kainz, E., \& Carbon, C. C. (2009). Recognition of emotional expressions is affected by inversion and presentation time. Perception, 38(12), 18491862. doi:10.1068/P6448

Ebner, N. C., Riediger, M., \& Lindenberger, U. (2010). FACES-A database of facial expressions in young, middle-aged, and older women and men: Development and validation. Behavior Research Methods, 42(1), 351-362. doi:10.3758/brm.42.1.351

Eisenbarth, H., \& Alpers, G. W. (2011). Happy mouth and sad eyes: scanning emotional facial expressions. Emotion, 11(4), 860-865. doi:10.1037/a0022758

Faul, F., Erdfelder, E., Lang, A.-G., \& Buchner, A. (2007). G*Power 3: A flexible statistical power analysis program for the social, behavioral, and biomedical sciences. Behavior Research Methods. doi:10.3758/BF03193146

Fischer, A. H., Gillebaart, M., Rotteveel, M., Becker, D., \& Vliek, M. (2012). Veiled emotions. Social Psychological and Personality Science, 3(3), 266-273. doi:10.1177/1948550611418534

Running title: The impact of face masks on emotional reading 
1 Golan, O., Baron-Cohen, S., \& Hill, J. (2006). The Cambridge mindreading (CAM) face-voice battery: Testing complex emotion recognition in adults with and without Asperger syndrome. Journal of Autism and Developmental Disorders, 36(2), 169-183. doi:10.1007/s10803-005-0057-y

Gosselin, F., \& Schyns, P. G. (2001). Bubbles: a technique to reveal the use of information in recognition tasks. Vision Research, 41(17), 2261-2271.

Grüter, T., \& Carbon, C. C. (2010). Escaping attention. Some cognitive disorders can be overlooked. Science, 328(5977), 435-436. doi:10.1126/science.1190432

Jefferson, T., Foxlee, R., Del Mar, C., Dooley, L., Ferroni, E., Hewak, B., . . Rivetti, A. (2008). Physical interventions to interrupt or reduce the spread of respiratory viruses: systematic review. BMJ (Clinical research ed.), 336(7635), 77-80. doi:10.1136/bmj.39393.510347.BE

Kotsia, I., Buciu, I., \& Pitas, I. (2008). An analysis of facial expression recognition under partial facial image occlusion. Image and Vision Computing, 26(7), 1052-1067. doi:10.1016/j.imavis.2007.11.004

Kret, M. E., \& de Gelder, B. (2012). Islamic headdress influences how emotion is recognized from the eyes. Frontiers in Psychology, 3(110), 1-13. doi:10.3389/fpsyg.2012.00110

Kuznetsova, A., Brockhoff, P. B., Rune, H. B., \& Christensen, A. P. \{lmerTest\} Package: Tests in linear mixed effects models. Journal of Statistical Software, 82(13), 1-26. doi:10.18637/jss.v082.i13

Leach, A.-M., Ammar, N., England, D. N., Remigio, L. M., Kleinberg, B., \& Verschuere, B. J. (2016). Less is more? Detecting lies in veiled witnesses. Law and Human Behavior, 40(4), 401-410. doi:10.1037/lhb0000189

Running title: The impact of face masks on emotional reading 
1 Mniszewski, S. M., Del Valle, S. Y., Priedhorsky, R., Hyman, J. M., \& Hickman, K. S. (2014). Understanding the impact of face mask usage through epidemic simulation of large social networks. In V. K. Mago \& V. Dabbaghian (Eds.), Theories and simulations of complex social systems (Vol. 52, pp. 97-115). Berlin: Springer.

Mondloch, C. J. (2012). Sad or fearful? The influence of body posture on adults' and children's perception of facial displays of emotion. Journal of Experimental Child Psychology, 111(2), 180-196. doi:10.1016/j.jecp.2011.08.003

Morey, R. D. (2008). Confidence intervals from normalized data: A correction to Cousineau (2005). Tutorials in Quantitative Methods for Psychology, 4(2), 61-64.

R Core Team. (2014). R: A language and environment for statistical computing. Retrieved from http://www.R-project.org/

Roberson, D., Kikutani, M., Doge, P., Whitaker, L., \& Majid, A. (2012). Shades of emotion: What the addition of sunglasses or masks to faces reveals about the development of facial expression processing. Cognition, 125(2), 195-206. doi:10.1016/j.cognition.2012.06.018

Rojahn, J., Gerhards, F., Matlock, S. T., \& Kroeger, T. L. (2000). Reliability and validity studies of the Facial Discrimination Task for emotion research. Psychiatry Research, 95(2), 169181.

Ruffman, T., Henry, J. D., Livingstone, V., \& Phillips, L. H. (2008). A meta-analytic review of emotion recognition and aging: implications for neuropsychological models of aging. Neuroscience and Biobehavioral Reviews, 32(4), 863-881. doi:10.1016/j.neubiorev.2008.01.001

Running title: The impact of face masks on emotional reading 
1 Russell, J. A. (1994). Is there universal recognition of emotion from facial expression: A review

van der Sande, M., Teunis, P., \& Sabel, R. (2008). Professional and home-made face masks reduce exposure to respiratory infections among the general population. PLoS ONE, 3(7), e2618. doi:10.1371/journal.pone.0002618

Wegrzyn, M., Vogt, M., Kireclioglu, B., Schneider, J., \& Kissler, J. (2017). Mapping the emotional face. How individual face parts contribute to successful emotion recognition. PLoS ONE, 12(5), e0177239. doi:10.1371/journal.pone.0177239

Willis, J., \& Todorov, A. (2006). First impressions: Making up your mind after a 100-ms exposure to a face. Psychological Science, 17(7), 592-598. doi:10.1111/j.14679280.2006.01750.x

Wu, Z., \& McGoogan, J. M. (2020). Characteristics of and important lessons from the Coronavirus Disease 2019 (COVID-19) outbreak in China: Summary of a report of 72314 cases from the Chinese Center for Disease Control and Prevention. JAMA, 323(13), 1239-1242. doi:10.1001/jama.2020.2648 